\title{
Relato de caso: Diabetes tipo LADA ou "Double Diabetes"? Nem todo diabetes é tipo 1 ou tipo 2
}

\author{
Case Report: LADA or Double Diabetes? Not every diabetes is type 1 or type 2
}

Aline Braga Soares ${ }^{\dagger}$ Guilherme Thomaz D. Dumay ${ }^{\dagger}$ Cintia Marques ${ }^{\ddagger}$, Vivian C. M. Ellinger*

Como citar esse artigo. Soares, $\mathrm{AB}$ Dumay, GTD; Marques, C; Ellinger, VCM. Relato de caso: Diabetes tipo LADA ou "Double Diabetes"? Nem todo diabetes é tipo 1 ou tipo 2. Revista de Saúde. 2017 Jul./Dez.; 08 (2): 03-06.

\section{Resumo}

O diabetes é uma doença muito prevalente e com múltiplas vias patogênicas. Diversos fenótipos compõem o espectro dessa doença, alguns clinicamente semelhantes, como o "Latent Autoimmune Diabetes of the Adult" (LADA) e o "Double Diabetes" (DD), dificultando o diagnóstico e o manejo clínico. Em ambas as apresentações, ocorre uma intersecção de características típicas do diabetes tipo 1 (DM1) e tipo 2 (DM2). Relata-se o caso de uma paciente de 44 anos, que iniciou um quadro de diabetes gestacional há 20 anos. Após dois anos sem tratamento, apresentou emagrecimento, polidipsia e diplopia, associadas ao teste anti-GAD (glutamic acid descarboxilase) positivo, sendo diagnosticada como DM1. Ao procurar o ambulatório a paciente apresentava quadro de síndrome metabólica. Este trabalho visa discutir o complexo diagnóstico do diabetes. Embora esta seja uma doença muito estudada, a cada nova evidência científica um antigo conceito cai em desuso e fica evidenciado o quanto a realização de estudos sobre o tema ainda é necessária.

Palavras-chave: Diabetes Mellitus; Diabetes Autoimune Latente do Adulto (LADA); Double Diabetes (DD); Classificação do Diabetes.

\begin{abstract}
Diabetes is a common endocrine disease with multiple pathogenic pathways. Several phenotypes make up the spectrum of this disease; some of them are clinically similar, such as Latent Autoimmune Diabetes of the Adult (LADA) and Double Diabetes (DD), hindering proper diagnosis and clinical management. There is an intersection of typical features of type 1 diabetes (DM1) and type 2 (DM2) in both presentations. We report the case of a 44-year-old female patient, who acquired gestational diabetes 20 years ago. After two years without treatment, she presented weight loss, polydipsia and diplopia, associated with an anti-GAD positive test, being diagnosed as DM1. The patient arrived at the outpatient clinic, demonstrating signs and symptoms of metabolic syndrome. This case report discusses the complex diagnosis of diabetes. Although this is a well-studied disease, with every new scientific evidence an old concept is abandoned and highlights the importance of more studies on diabetes.
\end{abstract}

Keywords: Diabetes Mellitus; Latent Autoimmune Diabetes of the Adult (LADA); Double Diabetes (DD); Diabetes Classification.

\section{Introdução}

A Diretriz da Sociedade Brasileira de Diabetes informa que "Diabetes Mellitus não é uma única doença, mas um grupo heterogêneo de distúrbios metabólicos que apresenta em comum a hiperglicemia, resultante de defeitos na ação da insulina, na secreção de insulina ou em ambas"(1). Dentre os diversos tipos de manifestações, está o Diabetes Autoimune Latente do Adulto (LADA) e o "Double Diabetes" (DD), que se confundem clinicamente. O LADA baseia-se na presença de anticorpos ácido glutâmico descarboxilase (anti-GAD) em pacientes com início de Diabetes Mellitus (DM) acima de 35 anos de idade e que são independentes de insulina por pelo menos seis meses após o diagnóstico (2). Estudos epidemiológicos sobre LADA esbarram em problemas metodológicos, visto que idade de início da doença e a necessidade precoce de insulina não são parâmetros adequados para definir uma patologia (3). A importância em se conhecer a epidemiologia do Diabetes pauta-se na atual estimativa de que a população mundial com diabetes seja na ordem

Afiliação dos autores: $\uparrow$ Discentes Universidade Severino Sombra, Discente do curso de Medicina- Vassouras/RJ.

$\$$ Docentes Universidade Severino Sombra, Docente do curso de Medicina- Vassouras/RJ Brasil. 
de 387 milhões e que alcance 471 milhões em 2035 (1). Em um interessante relato de caso, Rapti define o LADA como uma forma lentamente progressiva de diabetes autoimune caracterizado por idade avançada no momento do diagnóstico em comparação com o DM1 (4). Ao mesmo tempo tem surgido um número crescente de crianças e jovens com uma combinação dos dois tipos principais de diabetes, isto é, indivíduos obesos e/ou com sinais de resistência à insulina associada à presença de marcadores de autoimunidade contra as células-beta, características que definem o DD. Este subgrupo, também pode ser chamado de "diabetes híbrido", "diabetes tipo 1,5" ou "diabetes autoimune latente do jovem (LADY)" (5) . O presente artigo teve como objetivo relatar um caso de diabetes imunomediado, além de ressaltar a importância de um tratamento eficaz. O adequado controle da glicemia, é indispensável, uma vez que previne as complicações macro e microvasculares. O trabalho visa também, secundariamente, dar enfoque à dificuldade de se obter $\mathrm{o}$ diagnóstico correto tendo em vista a atual classificação, quando não se trata de um caso clássico de DM1 ou DM2.

\section{Relato do caso}

Uma paciente do sexo feminino, caucasiana, de 44 anos de idade, com história familiar paterna de DM2 e história pessoal de diabetes gestacional há 20 anos, sem tratamento na ocasião, com histórico de hipotireoidismo, diagnosticado na adolescência, o qual se manteve controlado com levotiroxina sódica (Puran T4®) $100 \mathrm{mg} 1 \mathrm{x} / \mathrm{dia}$, relatou ter procurado o ambulatório de endocrinologia pela primeira vez em 1998, aos 26 anos, frente a um quadro de perda ponderal, diplopia e polidipsia, sendo diagnosticada com DM1. Naquela primeira consulta, foi instituído tratamento com insulina regular e insulina NPH (Neutral Protamine Hagedorn). Este esquema terapêutico foi mantido por 17 anos sem adequado controle, devido à pouca assiduidade da paciente às consultas ambulatoriais. Em agosto de 2015, quando a paciente retornou às consultas rotineiras, foi identificada dislipidemia e iniciado o tratamento com sinvastatina $(20 \mathrm{mg} / \mathrm{dia})$.

Os exames laboratoriais daquele período revelaram também: hemoglobina glicada $(\mathrm{HbA} 1 \mathrm{c})$ $10,2 \%$ e glicemia de jejum de $246 \mathrm{mg} / \mathrm{dl}$, indicando descontrole glicêmico, optando-se pela troca do esquema insulínico anterior por insulina glargina (Lantus $\left.{ }^{\circledR}\right)$, com intuito de manter uma liberação basal de insulina, associado à insulina aspart (Novorapid®), 15 minutos antes das principais refeições. Além disso, foi solicitado que a paciente fizesse monitoração glicêmica capilar domiciliar de quatro em quatro horas, visando guiar as intervenções subsequentes. O quadro clínico vinha em adequado controle até novembro de 2015, quando a paciente apresentou picos de hiperglicemia frequentes e episódios sintomáticos de hipoglicemia esporadicamente durante a semana. Negava períodos de jejum prolongados. Nesta época, foram solicitados novos exames laboratoriais $(\mathrm{HbAlc}$, glicemia de jejum e anti-GAD), tendo sido adicionado ao esquema terapêutico a metformina $(1500 \mathrm{mg} /$ dia) e a contagem de carboidratos com intuito de guiar com melhor precisão a dosagem insulínica. Em março de 2016, a paciente trazia consigo os seguintes resultados de exames laboratoriais, solicitados na consulta anterior: $\mathrm{HbA} 1 \mathrm{c}$ de 7.6\%; glicemia de jejum de $177 \mathrm{mg} / \mathrm{dl}$ e anti-GAD (positivo) de 159,6 U/ml. Ao exame físico verificou-se índice de massa corporal (IMC) de 26,7, demonstrando sobrepeso, e ausência de acanthosis nigricans. A paciente apresentava síndrome metabólica de acordo com os critérios da International Diabetes Federation (IDF) (6) evidenciados através da observação de obesidade central (circunferência abdominal de $88 \mathrm{~cm}$ ), dislipidemia em tratamento com sinvastatina e glicemia de jejum de $177 \mathrm{mg} / \mathrm{dl}$. Nesta mesma consulta, foi solicitada nova dosagem de HbA1c, cujo resultado foi $6,7 \%$. De acordo com a evolução clínica, fica clara a melhora da paciente com as intervenções adotadas e, portanto, seu diagnostico foi revisto e enquadrado como diabetes imunomediado associado à fatores de resistência insulínica, sendo os dois principais diagnósticos diferenciais para o caso LADA e DD.

\section{Discussão}

LADA e DD são manifestações com perfil genético e imunológico semelhante ao do DM1, mas com características clínicas em comum com o DM2. Esta paciente, com histórico de DM gestacional, permaneceu sem tratamento por dois anos e obteve diagnóstico inicial de DM1. Todavia, de acordo com os critérios da IDF, desenvolveu síndrome metabólica (SM), o que não é habitual neste fenótipo. Azambuja, afirma que "ficou muito evidente a obrigatoriedade da medida da circunferência abdominal, representativa de obesidade central, facilitando a inclusão dos sujeitos no diagnóstico de SM" (6). Outro ponto a ser considerado, é a sugestão de Dib (2006), em que o tratamento insulínico intensivo pôde desmascarar a obesidade central ou a SM em um grupo de indivíduos com DM1 (7). Entretanto, nesta época, a prevalência e o significado da SM eram menos estudados nesse grupo de pacientes com autoimunidade presente, de forma que o DD não foi considerado no estudo em questão. Em um estudo mais recente, este mesmo autor expõe que o aumento da prevalência de obesidade associado ao sedentarismo e o diagnóstico precoce da presença 
de autoimunidade contra células beta, tem possibilitado o aparecimento de uma intersecção entre os dois tipos mais frequentes de Diabetes (DM1 e DM2) no mesmo indivíduo, caracterizando DD (5).

Resumidamente pode-se visualizar na Tabela 1 o contraste entre os parâmetros clínicos utilizados no

Tabela 1. Parâmetros clínicos para o diagnóstico de LADA e DD.

\begin{tabular}{ccc}
\hline Parâmetro & LADA & DD \\
\hline Início do DM & $>35$ anos & Jovem $(<35)$ \\
IMC & $<25 \mathrm{~kg} / \mathrm{m}^{2}$ & $>25 \mathrm{~kg} / \mathrm{m}^{2}$ \\
\hline $\begin{array}{c}\text { Histórico doença } \\
\text { autoimune }\end{array}$ & Presente & Ausente \\
Anti-GAD & Positivo & Positivo \\
& & \\
SM & Ausente & Presente \\
\hline
\end{tabular}

Fontes: Silva M, 2007(3) e Wilkin TJ, 2001(8)

diagnóstico de LADA e DD.

A paciente deste caso apresentou teste anti-GAD positivo, característica comum tanto ao LADA como ao DD, além de apresentar clínica inicial aos 24 anos, IMC $>25(26,7) \mathrm{e} \mathrm{SM}$, estes últimos aspectos mais compatíveis com DD. Contudo, existe uma frequente associação entre LADA e a autoimunidade tireoidiana (3), o que a aproxima do subgrupo LADA. Quando o quadro da paciente e a sua evolução foram considerados, a paciente foi diagnosticada com DD. A literatura relata o caso de um paciente diagnosticado com LADA, apesar dos 31 anos, apresentando um quadro extremamente semelhante ao desta paciente: hiperglicemia $(300 \mathrm{mg} / \mathrm{dl})$, poliúria, polidipsia, perda de peso e "visão embaçada" e que devido à recusa veemente de ser tratado com insulina, foi tratado com uma terapia alternativa com inibidor da dipeptil peptidase (Sitagliptina ${ }^{\circledR}$ ) e vitamina D, pois havia hipovitaminose, obtendo-se excelente controle glicêmico dentro de oito semanas, o que persistiu por dois anos de acompanhamento (4). Relatos como este motivam a terapêutica centrada no reconhecimento das vias produtoras de hiperglicemia em cada paciente e reforçam a necessidade de uma classificação mais útil para a Diabetes, na prática médica.

Existem diversas teorias na tentativa de determinar a relação entre DM1 e DM2. Uma das principais propostas citada pela comunidade científica é a hipótese aceleradora $(5,8,9)$. Esta hipótese identifica fatores que aceleram a destruição da massa de células beta-pancreáticas como de origem constitucional (genes e gestação), de resistência à insulina (lipo e glicotoxicidades), e imunológicos (sistema HLA) (8). Nesta hipótese, a sobrecarga imposta às células beta devido à resistência insulínica as deixa mais vulneráveis ao ataque do sistema imune. Isso poderia justificar faixas etárias intermediárias de aparecimento clínico do Diabetes, nos casos em que a autoimunidade e a resistência insulínica estiverem associadas na fisiopatologia. Acredita-se que a sobreposição entre os fenótipos DD e DM2 no jovem já esteja bem evidenciada, ao contrário do que acontece na população adulta em relação ao LADA e DM2 (5). Admite-se que o LADA pode corresponder à aproximadamente $10 \%$ dos casos de DM2, mas a resistência à insulina e a obesidade não estão entre as principais características dos indivíduos com LADA, como ocorre no DD (6). Tendo em vista que a paciente estudada melhorou clinicamente após o uso de metformina, responsável pela diminuição da resistência insulínica, infere-se que esta seja uma via patogênica importante na gênese de sua hiperglicemia, além da autoimunidade, favorecendo o diagnóstico para DD.

$\mathrm{O}$ atual sistema de classificação apresenta desafios para o diagnóstico e tratamento de pacientes com DM. Uma das novas propostas de classificação é centrada em células beta, englobando todos os subtipos de DM conhecidos, apoiando melhores práticas em gestão desta patologia através da identificação das vias que promovem a hiperglicemia, dirigindo o tratamento para disfunções específicas e com relação à terapêutica, as sulfonilureias têm mostrado a indução de apoptose em cultura de células beta (2). Em contrapartida, há relatos de uma melhoria significativa da resposta das células beta com o tratamento à base de inibidores de DPP4, entre outras drogas mais modernas (4). Essas evidências denotam que a situação relativa ao tratamento medicamentoso também precisa ser revista.

\section{Conclusão}

O caso relatado exemplifica a dificuldade diagnóstica na prática médica, uma vez que alguns pacientes não apresentam uma clínica que permita o diagnóstico claro de DM1 ou DM2, fazendo com que os tipos mistos de diabetes sejam considerados com mais frequência, o que influencia o tipo de tratamento adequado. Há a possibilidade de que a hiperinsulinemia, ocasionada à introdução precoce de insulinoterapia, esteja associada a um risco aumentado de doença cardiovascular, uma vez que pode induzir à obesidade central devido ao efeito anabólico da medicação (6). Como discutido anteriormente, a vigente classificação do diabetes não atende adequadamente ao atual cenário com múltiplas apresentações clínicas "atípicas" e sobrepostas do DM. Até que meios mais práticos e 
claros para classificar o diabetes com seus variados subtipos sejam desenvolvidos, é importante tratar de forma personalizada os pacientes com base em sua manifestação clínica e características bioquímicas individuais. Dessa forma, é essencial identificar a via responsável pela gênese da hiperglicemia com intuito de aperfeiçoar o manejo clínico e as chances de sucesso terapêutico.

\section{Referências}

1. SBD SBdD. Diretrizes da Sociedade Brasileira de Diabetes. 2012. Diabetes. 2015; 1 .

2. Schwartz SS, Epstein S, Corkey BE, Grant SF, Gavin JR, Aguilar RB. The time is right for a new classification system for diabetes: rationale and implications of the $\beta$-cell-centric classification schema. Diabetes Care. 2016;39(2):179-86.

3. Silva MERd. Precisamos diagnosticar o diabetes Latente Autoimune do Adulto (LADA)? Arquivos Brasileiros de Endocrinologia \& Metabologia. 2007;51(1):8-10.

4. Rapti E, Karras S, Grammatiki M, Mousiolis A, Tsekmekidou X, Potolidis E, et al. Combined treatment with sitagliptin and vitamin D in a patient with latent autoimmune diabetes in adults. Endocrinology, diabetes \& metabolism case reports. 2016;2016.

5. Dib SA. Heterogeneidade do diabetes melito tipo 1. Arquivos Brasileiros de Endocrinologia \& Metabologia. 2008.

6. Azambuja CR, Farinha JB, Rossi DS, Spohr CF, Santos DLd. O Diagnóstico da síndrome metabólica analisado sob diferentes critérios de definição. Revista Baiana de Saúde Pública. 2015;39(3):482.

7. Dib SA. Resistência à insulina e síndrome metabólica no diabetes melito do tipo 1. Arquivos Brasileiros de Endocrinologia \& Metabologia. 2006.

8. Wilkin T. The accelerator hypothesis: weight gain as the missing link between type I and type II diabetes. Diabetologia. 2001;44(7):914-22.

9. Pozzilli P, Buzzetti R. A new expression of diabetes: double diabetes. Trends in Endocrinology \& Metabolism. 2007;18(2):52-7. 